\title{
Palliative Biliary Drainage Using Endoscopic Ultrasound-Guided Rendezvous Procedure by Transgastric Approach
}

\author{
Tiago Leal Pedro Antunes Sofia da Silva Mendes Raquel Gonçalves \\ Bruno Gonçalves \\ Gastroenterology Department, Hospital de Braga, Braga, Portugal
}

\author{
Keywords \\ Biliary drainage $\cdot$ Palliation $\cdot$ Rendezvous $\cdot$ Endoscopic \\ ultrasound
}

\section{Drenagem Biliar Paliativa através da Técnica de Rendez-vous Transgástrica guiada por Eco-endoscopia}

\section{Palavras Chave \\ Drenagem biliar · Paliação · Rendez-vous · \\ Eco-endoscopia}

We present a case of a 65 -year-old female patient, diagnosed with adenocarcinoma of the pancreatic head with liver metastasis. She underwent ERCP for biliary drainage using an uncovered self-expandable metal stent (SEMS; $6 \mathrm{~cm} \times 10 \mathrm{~mm}$, WallFlex ${ }^{\mathrm{TM}}$ Biliary RX Uncovered, Boston Scientific, Marlborough, MA, USA), followed by palliative chemotherapy. Eighteen months after the first procedure the patient presented to the emergency department where she was diagnosed with cholangitis and was admitted with IV antibiotics. A second ERCP was scheduled where the

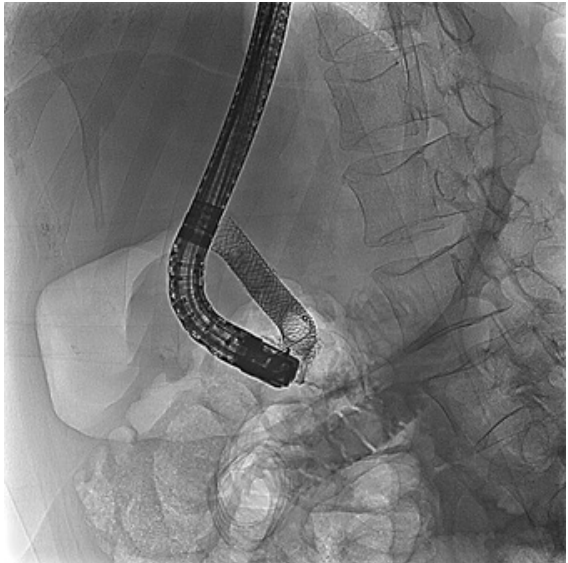

Fig. 1. Occlusion cholangiogram showing no contrast passage through the proximal end of the previously placed uncovered metal stent.

sphincterotome was easily introduced within the biliary metal stent but due to tumor ingrowth and overgrowth the guidewire $\left(0.035 \mathrm{in} \times 450 \mathrm{~cm}\right.$, Jagwire ${ }^{\mathrm{TM}}$, Boston Scientific, Marlborough, MA, USA) could not transverse the stenosis, despite multiple attempts, and there was no contrast passage through stent's proximal end (Fig. 1).

\section{(c) 2021 Sociedade Portuguesa de Gastrenterologia} Published by S. Karger AG, Basel

This is an Open Access article licensed under the Creative Common Attribution-NonCommercial-4.0 International License (CC BY-NC) (http://www.karger.com/Services/OpenAccessLicense), applicable to the online version of the article only. Usage and distribution for commercial purposes requires written permission.
Correspondence to:

Tiago Leal, tiago.afleal@gmail.com 

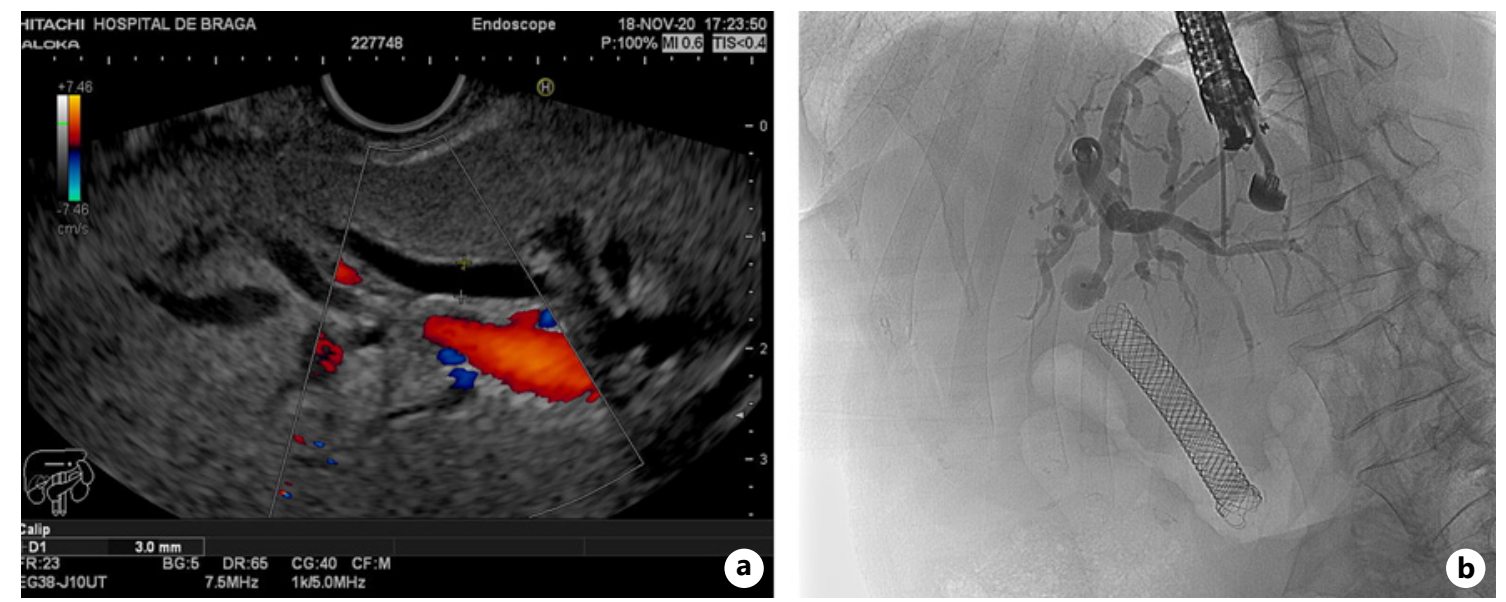

Fig. 2. a Endoscopic ultrasound with color Doppler showing a dilated left intrahepatic duct. b Cholangiography after contrast injection through the fine needle aspiration needle.

Fig. 3. a Fluoroscopic image showing the guidewire crossing the papilla after anterograde introduction. b Retrieval of the guidewire tip. c Duodenoscope adjacent to the metal stent after over-the-wire introduction. d Deployment of the uncovered self-expandable metal stent using the "stent-in-stent" technique.
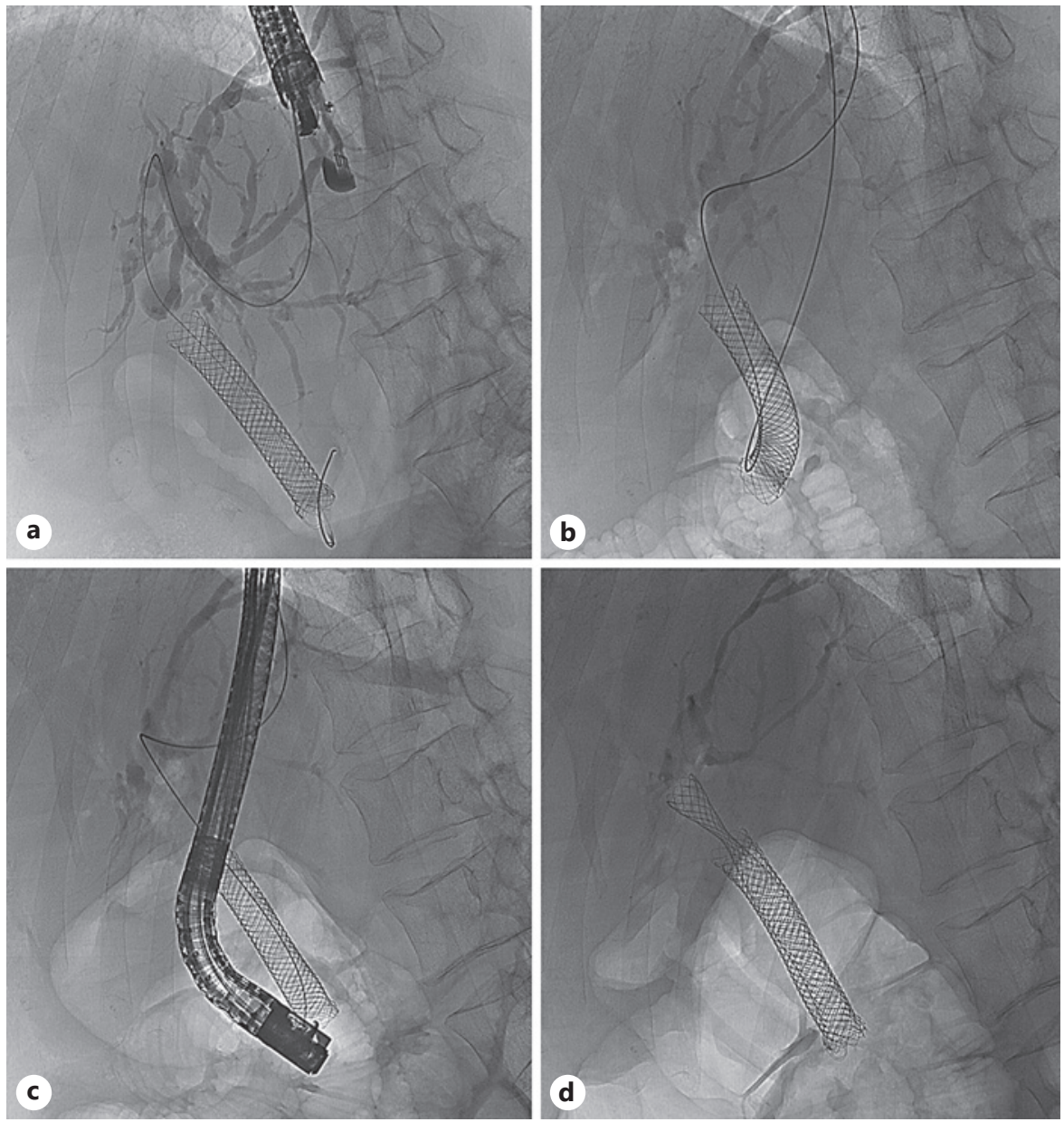
We then decided to try to achieve deep biliary cannulation using the rendezvous technique. A linear Pentax EG38-J10UT echoendoscope (Pentax medical, Tokyo, Japan) with Hitachi-Aloka HI VISION Noblus processor was used to identify the best biliary access, which was found to be transgastric. Color Doppler helped distinguish blood vessels from the dilated left intrahepatic biliary duct (Fig. 2a). A 3-mm-diameter hepatic duct was punctured by a 19-gauge fine needle aspiration needle (Expect $^{\mathrm{TM}}$ 19-gauge flex, Boston Scientific, Marlborough, MA, USA), followed by bile aspiration, confirming the correct intraductal positioning. Cholangiography was performed by injection of contrast medium through the needle (Fig. 2b). Afterwards, a guidewire was introduced in an anterograde fashion and passed all the way across the papilla into the duodenum (Fig. 3a). The echoendoscope was then removed and the guidewire tip was retrieved using a rat-tooth grasping forceps (Fig. 3b). Next a TJF-Q180V duodenoscope (Olympus Corporation, Tokyo, Japan) was introduced through the wire until the distal end of the previously placed SEMS was reached (Fig. 3c). A new SEMS $\left(6 \mathrm{~cm} \times 10 \mathrm{~mm}\right.$, WallFlex ${ }^{\mathrm{TM}}$ Biliary RX Uncovered, Boston Scientific, Marlborough, MA, USA) was deployed using the "stent-in-stent" technique (Fig. $3 \mathrm{~d}$ ), providing adequate biliary drainage. The patient was discharged the next day.

There are several options available when conventional deep biliary cannulation fails [1]. In this specific case, a slightly different problem arose, which was the inability to pass the guidewire through the biliary stenosis. Frequent strategies to overcome this problem consist in changing the guidewire, using a fully hydrophilic or an angled tip. Additionally, cholangioscopy could have been performed to assist the insertion of the guidewire [2], although availability and costs are important issues to con- sider. Finally, the fact that there was already a metal stent in situ hindered the employment of a duodenal approach for EUS-guided biliary drainage. Rendezvous procedure has been described as a salvage technique, reporting success rates around $74-80 \%$ and complication rates between 11 and 15\% [3, 4]. Although it requires an experienced therapeutic ultrasonographer, EUS-guided biliary drainage has the advantage of allowing a single-operator, one-stage procedure, when compared to percutaneous transhepatic cholangiography-assisted rendezvous [5].

\section{Statement of Ethics}

This research was conducted ethically in accordance with the World Medical Association Declaration of Helsinki. The patient gave written informed consent to publish this case.

\section{Conflict of Interest Statement}

The authors have no conflicts of interest to declare.

\section{Funding Sources}

There are no funding sources to declare.

\section{Author Contributions}

T.L. was responsible for the design of the study, collecting the data, and drafting of the manuscript. P.A. and S.S.M. helped collecting the data and drafting of the manuscript. R.G. and B.G. were responsible for the design of the study, interpretation of the data, and critical revision of the work for important intellectual content. All authors approved the final version to be published and agreed to be accountable for all aspects of the work.
References
1 Liao WC, Angsuwatcharakon P, Isayama $\mathrm{H}$, Dhir V, Devereaux B, Khor CJ, et al. International consensus recommendations for difficult biliary access. Gastrointest Endosc. 2017 Feb;85(2):295-304.

2 Parsi MA, Guardino J, Vargo JJ. Peroral cholangioscopy-guided stricture therapy in living donor liver transplantation. Liver Transpl. 2009 Feb;15(2):263-5.

3 Isayama H, Nakai Y, Kawakubo K, Kawakami $\mathrm{H}$, Itoi T, Yamamoto N, et al. The endoscopic ultrasonography-guided rendezvous tech- nique for biliary cannulation: a technical review. J Hepatobiliary Pancreat Sci. 2013 Apr; 20(4):413-20.

4 Iwashita T, Yasuda I, Mukai T, Iwata K, Ando N, Doi S, et al. EUS-guided rendezvous for difficult biliary cannulation using a standardized algorithm: a multicenter prospective pilot study (with videos). Gastrointest Endosc. 2016 Feb;83(2):394-400.

5 Itoi T, Dhir V. EUS-guided biliary rendezvous: slow, hesitant, baby steps forward. Gastrointest Endosc. 2016 Feb;83(2):401-3. 